\title{
Contraception and sexual health in obese women
}

\author{
Katharine B Simmons, MDa1, Alison B Edelman, MD MPH \\ ${ }^{a}$ Oregon Health \& Science University \\ Department of Obstetrics and Gynecology \\ 3181 SW Sam Jackson Park Road, Mailcode UHN 50 \\ Portland, OR 97239, USA \\ ${ }^{1}$ Corresponding author: \\ simmonka@ohsu.edu
}

$503-494-2811$

Conflict of Interest: Simmons: none. Edelman: consultant for Gynuity Health Projects, World Health Organization, Genzyme, and Agile Therapeutics. Nexplanon trainer for Merck. Author for UptoDate (Royalties received). She receives research funding from the National Institute of Health, USAIDs and the Bill \& Melinda Gates Foundation. These potential conflicts of interest have been reviewed and managed by OHSU. 


\section{Abstract}

As the proportion of women with obesity increases worldwide, understanding the influence of body weight on sexual behavior, fertility, and contraceptive effectiveness is critical for health care professionals and patients. Although many have theorized that obese women are different from normal weight women regarding sexual health and behavior, current evidence for the most part disproves this. The exception is in adolescents where body image may play a role in riskier behavior, placing them at greater risk for an unintended pregnancy. Given that most modern contraceptives were not originally evaluated in obese women, understanding how weight affects contraceptive pharmacokinetics and efficacy should be a focus of ongoing research. Evidence is reassuring that most modern contraceptive methods are safe and effective in obese women. This paper will review what is known about sexual and contraceptive behavior, as well as effectiveness and pharmacokinetics of modern contraceptives, for overweight and obese women.

Key words: obesity, contraception, sexual behavior, pharmacokinetics 


\section{Introduction}

Obesity is a worldwide epidemic, affecting 34\% of reproductive age women in the United States and at least $12 \%$ in Western Europe [1,2]. Obesity also affects women in less developed nations, especially in urban areas. The World Health Organization defines obesity as a body mass index over $30 \mathrm{~kg} / \mathrm{m}^{2}$, and worldwide, over 300 million women can be classified as obese [2,3]. Obesity is now the fifth leading cause of mortality worldwide[2].

For decades, contraceptive research excluded women over $130 \%$ of ideal body weight, utilizing a one-size fits all approach. However, obesity has the potential to influence fertility and contraceptive efficacy in several ways, including changes in baseline fecundity, sexual behavior, contraceptive use and steroid hormone pharmacokinetics. Obese women who become pregnant face an increased risk of gestational hypertension, diabetes, preeclampsia, Cesarean delivery, and fetal complications such as growth restriction, neural tube defects, and stillbirth [4,5]. It is a public health necessity to understand the safety and effectiveness of contraception in obese women to assist women and their health care providers in making informed choices about contraceptive use and childbearing.

This article will review what is known about the sexual and contraceptive behavior of obese women, as well as effectiveness and pharmacokinetics of modern contraceptives for overweight and obese women.

\section{Sexual health and behavior}


The probability of pregnancy is dependent on several factors, including frequency and timing of intercourse, baseline fecundity, and use of contraception. Body habitus has the potential to affect a woman's pregnancy risk through each of these mechanisms.

\section{Sexual behavior}

Body habitus and self-image may influence sexual and other behaviors. Though several large population-based studies have examined the association between BMI and sexual behavior, these studies are limited by self-report of very personal questions, and misreporting is common [6].

Adolescents appear to have greater differences in sexual behavior by body weight than adult women. In an analysis of data from the 2011 National Longitudinal Survey of Youth (NLSY), obese white adolescent girls were more likely than non-obese girls to have a partner at least three years older (59\% obese vs $42 \%$ non-obese, $\mathrm{p}<0.05$ ), more likely to have more than three sexual partners in one year (48 vs $28 \%$ non-obese, $\mathrm{p}<0.05$ ), and less likely to use condoms during their most recent sexual encounter ( $30 \%$ obese vs $62 \%$ non-obese, $\mathrm{p}<0.05$ ) [7]. These differences were not present among black adolescent obese females. A similar analysis combining NLSY data from 2005-2011 had similar findings, including an elevated odds of having multiple sexual partners in obese girls compared to normal weight girls (OR 1.59, 95\% CI 1.32-1.91), and an elevated risk of not using condoms (OR 1.3, 95\% CI 1.07-1.57) [8]. Obese girls were also at elevated odds of having 
coital debut before the age of 13 (OR 2.12, 95\% CI 1.6-2.81), and a history of sexual abuse or non-consensual sex (OR 1.37 (95\% CI 1.12-1.67).

Data on adult women is available from several surveys in the US and Europe. In the French National Survey of sexual behaviors (Contexe de la Sexualite en France), obese women were $30 \%$ less likely to report having a sexual partner in the past 12 months, and were less likely to have an occasional sex partner [9]. The likelihood of reporting more than one sexual partner in the past year decreased as BMI increased $(\mathrm{p}=0.003)$. Among women with a sexual partner, there was no difference in the frequency of sexual intercourse by BMI.

In a US population, using data from the 1999-2000 NHANES survey, obese women reported fewer sexual partners in the past year than normal weight women (mean 0.93 vs $1.15, \mathrm{p}<0.05$, $t$ test). There was no difference in lifetime number of partners by BMI (mean 5.24 normal weight, 4.77 overweight, 5.76 obese, $p>0.05$ for both comparisons) [6]. Similarly, an analysis of the 2002 NSFG also demonstrated no difference in frequency of sexual intercourse, number of current partners, age at first intercourse, number of partners in the past year, and number of lifetime partners between obese and normal weight women [10]. This lack of association persisted after adjusting for age, race/ethnicity, education, household income, gravidity, parity, cohabitation status, general health, and place of residence. There was no difference in sexual orientation with $9 \%$ of normal weight women, $10.1 \%$ of overweight women and $9.7 \%$ of obese women reporting being something other than heterosexual. 


\section{Fecundity}

The probability of conceiving a pregnancy during a single menstrual cycle is known as fecundity. Baseline fecundity may be altered in obese women due to ovulatory dysfunction, irregular menstrual cycles, and other vascular or inflammatory mechanisms that are incompletely understood [11]. Increasing BMI has been associated with an increased time to conception regardless of cycle regularity. In the prospective Collaborative Perinatal Project in the United States, probability of conceiving in a single cycle was reduced $18 \%$ for obese women compared to normal weight women, which translated into a median time to conception of 5 months for obese women compared to 3 months for normal weight women[11]. Reduction in fecundity persisted after adjusting for age, smoking, race, education, occupation and study center. In a population of subfertile ovulatory women undergoing infertility workup, probability of pregnancy decreased by $5 \%$ for every unit of BMI over $29 \mathrm{~kg} / \mathrm{m}^{2}$ [12]. Factors contributing to lower fecundity in obese women may be reversible, as weight loss in obese women has been shown to improve pregnancy rates and live birth weights in a population undergoing assisted reproductive technology [13].

\section{Use of any method of contraception}

Women are considered at risk of unintended pregnancy if they are able to become pregnant, sexually active with a male partner, and not currently desiring conception. Most recent data from the US National Survey of Family Growth (NSFG 2006-2010) estimates that $11 \%$ of women at risk of unintended pregnancy are not 
currently using any method of contraception [14]. Data from the United Nations Population Division estimates that use of any method of contraception among European women ranges from $81.0 \%$ in Northern Europe to $62.1 \%$ in southern Europe, though these figures apply to all women, not just those at risk of unintended pregnancy [15].

Use of contraception by overweight and obese women does not appear to differ from normal weight women. Within the US, the 2002 NSFG demonstrated that the odds of contraceptive nonuse was not significantly different for obese and normal weight women after adjusting for age, ethnicity, education, and pregnancy desire, with $28.0 \%$ of normal weight women, $25.2 \%$ of overweight women, and 25.3-33.0\% of obese women reporting use of no method of contraception [16]. Likewise, in a secondary analysis of the 2006 Behavioral Risk Factor Surveillance System (BRFSS) family planning module, use of contraception was not associated with BMI after controlling for age, race, education, income, parity, and marital status[17].

A large 2006 French random probability telephone survey including 5535 women (Contexte de la Sexualité en France, CSF) demonstrated the same lack of association; the odds of contraceptive nonuse for obese compared to normal weight women was not significant for any age group (OR 2.4, 95\% CI 0.69-8.93 for women 18-29 years, and $1.18,95 \%$ CI $0.5-2.81$ for women $30-49$ years) [9]. This study excluded pregnant women but was not restricted to women at risk of unintended pregnancy. 


\section{Contraceptive use by method}

Studies of contraceptive method choice by BMI status are conflicting, and comparisons between studies are made difficult by inconsistent inclusion of women not at risk of unintended pregnancy (such as women with prior sterilization). Most studies do not demonstrate an association between contraceptive method choice and BMI.

Using data from the 2002 NSFG, Kaneshiro et al grouped contraceptive methods into most effective (permanent and long acting reversible), effective (short acting hormonal methods), and least effective (non-prescription) [10]. There was no statistically significant association between BMI and level of contraceptive method effectiveness.

More recently, Callegari et al examined a subset of 1345 obese women at risk of unintended pregnancy from the 2006-2010 NSFG[18]. Of these, $21.5 \%$ reported no contraceptive use in the past one month, and $31.3 \%$ primarily used nonprescription methods including condoms, withdrawal and fertility awareness. Using the same database but including women of all BMIs, $11 \%$ of women at risk of unintended pregnancy used no method of contraception and $22.8 \%$ used nonprescription methods. No formal comparisons were performed between obese and normal weight women making it difficult to draw conclusions from this more recent data.

In the French CSF study, obese women under 30 were more likely to rely on less effective methods such as withdrawal compared to normal weight women (OR 8.51, 95\% CI 2.02 to 35.88), but no other differences in contraceptive use were 
noted by BMI category [9]. Finally, using the BRFSS database in the United States, the most popular method by both normal weight and overweight/obese women was the pill (34.6\% normal weight, $28.5 \%$ overweight, $25.8 \%$ obese). Use of procedural methods (tubal ligation, vasectomy, implants and IUDs) increased with BMI, with $50.5 \%$ of obese women utilizing these most effective methods compared to $36.2 \%$ of normal weight and $45.8 \%$ of overweight women $(p=0.0001)$ [17].

\section{Contraceptive adherence}

Effectiveness of short-acting methods of contraception is dependent on consistent use [19]. Social factors such as economic status, housing stability, employment, and education may influence a woman's ability to consistently access and utilize contraception. Westhoff et al noted that non-adherence with an oral contraceptive regimen was associated with residential poverty[20]. After adjusting for economic status, obesity and race/ethnicity also remained associated with inconsistent OCP use. Obesity is associated with female poverty in the United States, and incomplete characterization of economic status may have led to residual confounding in this study.

\section{Unintended pregnancy}

In large surveys such as the NSFG, pregnancy intention is generally coded as a dichotomous variable (e.g. yes/no). Classifying a pregnancy as planned or unplanned may be overly simplistic and can contribute to inaccurate reporting. Use of abortion history as a surrogate outcome is also problematic given that about half 
of unintended pregnancies are carried to term [21]. Despite these limitations, some studies have addressed rates of unintended pregnancies in obese versus non-obese women.

Using data from the 2002 NSFG, there was no association between unintended pregnancy within the past 5 years and BMI $(\mathrm{p}=0.62)$, and this lack of association held when history of abortion was used as the outcome [22]. A similar analysis utilizing more recent NSFG data has not yet been completed.

By contrast, in the French CSF study, obese women under 30 were four times more likely to report a prior unintended pregnancy or abortion than normal weight women, but this association was not present for women over 30 . The likelihood of reporting an abortion significantly increased with BMI among women under 30 $(p=0.035)$, as did the likelihood of unintended pregnancy $(p=0.001)$ [9]. The difference between these studies may be related to the age groups studied; the NSFG data was not analyzed by age in relation to unintended pregnancy.

\section{Summary}

Although many have theorized that obese women are different from their normal BMI counterparts regarding sexual health and behavior, current evidence for the most part disproves this. The one exception is in young women where body image may play a role in riskier behavior placing them at greater risk for an unintended pregnancy. 


\section{Effectiveness and pharmacokinetics of modern contraceptives in obese women}

Proving differences between obese and normal BMI women in the inherent efficacy of a contraceptive method can be difficult as the overall effectiveness of a method, especially shorter-acting methods, are highly influenced by user adherence. Though most initial contraceptive clinical trials excluded obese women, interval studies have helped provide reassurance that most modern methods of contraception are safe and effective in obese women. Accordingly, the World Health Organization Medical Eligibility Criteria for Contraceptive Use gives a category 1 (no restriction) or category 2 (benefits outweigh risks) to most methods for obesity (table 1). Providers and women can be reassured that otherwise healthy obese women can use most methods without concern for differences in contraceptive effect or side effects.

\section{Permanent contraception}

Permanent female contraception is one of the most commonly used methods of birth control, with about one in three women worldwide relying on tubal occlusion [23]. Over 600,000 surgical sterilization procedures are performed each year in the United States, with 30,000 in France [24]. Whereas in Europe, most surgical sterilization procedures are performed in women over 40, in the United States and elsewhere in the world, younger women who have completed childbearing choose these methods. As female sterilization relies upon physical 
occlusion or removal of fallopian tubes, there is little biologic plausibility for a decrease in efficacy in obese women. However, performing these procedures can be more difficult in obese women, and they do not confer the same noncontraceptive benefits of some hormonal methods. Vasectomy remains a more effective and less invasive option, and for women with a single reliable partner this can be a reasonable option [25]. Concurrent tubal sterilization at the time of bariatric surgery is another option for obese women not desiring future childbearing.

\section{Postpartum tubal sterilization}

Postpartum tubal ligation is performed at the time of Cesarean delivery or via mini-laparotomy following a vaginal delivery. The largest prospective study of sterilization failure rates is still the United States Collaborative Review of Sterilization (CREST), performed between 1978 and 1987. This study indicated that postpartum sterilization via partial salpingectomy had the lowest failure rate over ten years, at 7.5 per 1000 women [26]. It may be difficult to exteriorize the uterus and tubes at the time of Cesarean delivery for obese women, making partial salpingectomy difficult. Use of Filshie clips can be considered in this scenario, though their failure rate is higher than that of partial salpingectomy $[0.017$ vs 0.004 over 2 years, respectively, $\mathrm{p}=0.04$ ] [27]. For women who deliver vaginally, minilaparotomy may be associated with higher complication rates including surgical site infection in obese women. Adequate retraction of subcutaneous tissue can help with visualization of the tubes; a small Alexis (Applied Medical, Racho Santa 
Margarita, California, USA) surgical retractor may aid in retraction and compression of subcutaneous tissue for obese women. All women should be counseled on the option of postpartum sterilization and long acting reversible contraceptives during pregnancy. Women unable to obtain postpartum tubal sterilization may be offered postplacental IUD or immediate postpartum contraceptive implant placement, which both offer similar level of effectiveness unlikely to be affected by BMI.

\section{Interval laparoscopic sterilization}

The most commonly performed method of interval laparoscopic sterilization is the Filshie clip, though bilateral salpingectomy is advocated by some as a possible ovarian cancer risk reduction strategy[28]. No studies since CREST have examined long term failure rates of interval sterilization procedures, and no weight analysis was performed of the CREST data. Failures rates are assumed to be the same in obese and non-obese women.

A secondary analysis examined complication rates by BMI with the original CREST data. This included 9475 obese women undergoing sterilization procedures. The rate of complications, including perioperative fever, transfusion, reoperation, rehospitalization, and conversion to laparotomy) was 1-2\%. Obesity did confer an elevated risk of complications (adjusted odds ratio 1.7 (95\% CI 1.2-2.6), as did diabetes, use of general anesthesia, and previous abdominal or pelvic surgery. of note, the interval sterilization procedures included in this data were performed under mini-laparotomy rather than laparoscopy, so these safety findings may not apply to modern laparoscopic approaches. 


\section{Hysteroscopic sterilization}

In 2002, the US FDA approved a nickel-titanium tubal microinsert coil (Essure, Conceptus, Mountainview, CA, USA) which was quickly adopted in Europe. This product allowed for sterilization transcervically, without abdominal surgery or general anesthesia. Hysteroscopic sterilization is considered a first line method in France [24], but long term efficacy studies are not available. Successful tubal occlusion with Essure relies upon correct placement of the inserts and a local tissue reaction leading to tubal scarring. There does not appear to be a higher risk of unsuccessful coil placement in obese compared to normal weight women [29]. However, failure to place both coils occurs in up to $10 \%$ of women, and up to $5 \%$ of women with bilateral coil placement will not develop tubal occlusion [29]. A recent decision analysis factoring in up to a $25 \%$ failure rate in coil placement estimated that 10 year failure rates would be significantly higher with hysteroscopic versus laparoscopic sterilization (96 per 1000 for hysteroscopic compared to 24-30 per 1000 for laparoscopic)[30]. When completing a hysteroscopic sterilization procedure on an obese patient, surgeons should consider ways to optimize visualization to maximize the potential of correct bilateral coil placement.

\section{Long acting reversible contraceptives}


Long acting reversible contraceptives, or LARCs, offer the most effective reversible options for preventing pregnancy and should be considered first line for all women regardless of body habitus.

\section{Intrauterine contraception}

There are currently several intrauterine systems available for contraception. These include the copper releasing devices such as the Copper T380, and progestinreleasing devices such as the levonorgestrel intrauterine system. The LNG-IUS was first approved in Europe in the early 1990s, and entered the US market in 2001. The LNG-IUS exerts its primary mechanism through a sterile inflammatory process, changes in cervical mucus, and suppression of the endometrium, whereas the copper IUD provides contraceptive effect by changing sperm and ova motility and creating a sterile inflammatory response. Ovulation suppression is not a primary mechanism of action of any IUD. Both types are highly effective methods of reversible contraception, with failure rates less than $1 \%$ per year, comparable to sterilization[19,25].

Contraceptive effectiveness of intrauterine contraception should be unaffected by body weight, since the mechanism of actions do not rely on ovulation suppression or systemic hormone levels. This theoretical equivalence is supported by clinical studies. The Contraceptive CHOICE project, which included nearly 6000 women including $27 \%$ of overweight and $35 \%$ obese, overall failures rates of IUDs were less than 1 per 100 woman-years, with no difference by BMI [31]. 


\section{Obesity-related issues}

One of the primary benefits of the LNG-IUS in all women is reduced menstrual bleeding. Obese women are more likely to experience abnormal uterine bleeding due to disruption of the hypothalamic-pituitary-ovarian axis. The LNG-IUS is approved in the US and Europe as a treatment for heavy menstrual bleeding. Normal and obese women experience similar reductions in bleeding and rates of amenorrhea, approximately $50 \%$ by two years of use [32]. Another major noncontraceptive benefit of intrauterine contraception for obese women is a reduction in the risk of endometrial cancer. Both the copper IUD and LNG IUD reduce this risk, which may be particularly relevant for women with oligo-ovulation or anovulation who are at higher risk of developing endometrial hyperplasia [33].

Neither IUD is associated with weight gain. However, women do experience weight gain with aging $(0.5-1 \mathrm{~kg} /$ year $)$, so long term users of the IUD may notice weight gain that is unrelated to the device $[34,35]$.

\section{Contraceptive Implants}

Progestin-releasing contraceptive implants currently available include the single-rod etonogestrel (ENG) implant (Nexplanon/Implanon, Merck, Whitehouse Station, NJ, USA), which is approved for three years of use, and a two-rod levonorgestrel (LNG) implant (Jadelle, Leiras Oy Pharmaceuticals, Turku, Finland) which provides contraceptive effect for five years.

The contraceptive implants are one of the most effective methods of reversible contraception regardless of weight. Original studies of ENG implant 
efficacy did not include women over $130 \%$ of ideal body weight, and no studies have directly addressed contraceptive efficacy in overweight or obese women. However, a secondary analysis of the contraceptive CHOICE project evaluated contraceptive failure among 1,168 implant users including 35\% who were obese and 28\% overweight[31]. The cumulative rate of implant failure over three years was 0.00 per 100 woman years in normal weight women, and 0.23 per 100 woman years in obese women. The one failure occurred in an obese woman four days after insertion and was likely an unrecognized luteal phase pregnancy rather than a method failure. This large prospective cohort study provides good support for excellent contraceptive effect in obese women for the full three years of use. Consistent with these findings, the UK and CDC MEC support unrestricted use of the ENG implant by obese women for the full duration of use (3 years) (table 1).

The two-rod LNG implant was originally studied in a population of women with a mean body weight of $62.4 \mathrm{~kg}$, including $25.8 \%$ of whom were over $70 \mathrm{~kg}$. Two on-treatment pregnancies occurred, in women weighing 65 and $80 \mathrm{~kg}$. The cumulative pregnancy rate over 5 years was 0.8 per 100 woman years and did not vary by weight category. The LNG implant is therefore considered effective for five years in women regardless of weight [36].

\section{Pharmacokinetics}

The ENG implant reaches peak serum concentration on day four after insertion, but is sufficient to suppress ovulation within 24 hours of insertion $[37,38]$. Serum concentrations gradually decline over time. Plasma concentrations 
of ENG are inversely related to body weight, and have been measured as much as 47.6\% lower in obese women than in normal weight women in one small study[37]. The threshold of ovulation suppression appears to be $90 \mathrm{pg} / \mathrm{ml}$ [39]. Serum levels of ENG may approach this threshold by three years in obese women, raising a theoretical concern for decrease in contraceptive effect. However, increasing failure rates in obese women at three years has not been clinically demonstrated, suggesting that other contraceptive properties such as cervical mucus effects may continue to provide contraceptive protection at lower serum levels.

The two-rod levonorgestrel implant reaches maximum serum concentrations after 24-72 hours, falls moderately for the next week, and then continues with a very gradual decline over 3-7 years [40]. Mean LNG concentration after one month of use was $435 \mathrm{pg} / \mathrm{ml}(+/-13)$. Plasma concentrations of levonorgestrel are consistently lower for women of higher body weight, but follow a similarly gradual decline. For all women, serum levels at 5 years were $63-68 \%$ of their initial values in each weight category. The threshold of contraceptive efficacy is considered to be 180pg/ml. Despite consistently lower plasma concentrations, mean LNG concentrations remain above $200 \mathrm{pg} / \mathrm{ml}$ for a full five years for women over $70 \mathrm{~kg}$.

\section{Obesity related issues}

Like other hormonal methods, perception of weight gain may be greater than actual weight gain with contraceptive implants. Though up to $12 \%$ of ENG implant users report weight gain, the actual documented mean weight gain over three years was 1.6kg in original studies of normal weight women [41-43]. Likewise, average 
weight change in users of the two-rod LNG implant was an increase $1.1 \mathrm{~kg}$ over five years [36]. Importantly, in the Contraceptive CHOICE project, there was no difference in weight gain between users of the ENG implant and copper IUD, suggesting that weight gain with long-term methods may be more related to age and lifestyle than a contraceptive side effect [35].

\section{Short acting reversible contraceptives}

\section{Depot Medroxyprogesterone Acetate}

Depot medroxyprogesterone acetate (DMPA) is a method of injectable contraception, administered every three months in an intramuscular form (150mg) or every one month in a subcutaneous form (104mg).. DMPA provides contraceptive effect primarily through ovulation suppression $[44,45]$, with a typical use failure rate of $6 \%$, and a perfect use failure rate of $0.2 \%$ over one year [25]. Ovulation suppression is equivalent in obese and thin women for 12 weeks after IM administration [46]. This pharmacodynamic equivalence has also been demonstrated in clinical studies, where there is no difference in IM DMPA failure rates in obese versus normal weight women [47]. Smaller studies of DMPA-SC also show no difference in ovulation or method failure between obese and normal weight women $[45,48]$.

\section{Pharmacokinetics}


DMPA can be detected in serum within 30 minutes of intramuscular administration, and reaches a threshold $(100 \mathrm{pg} / \mathrm{ml})$ to suppress ovulation within 24 hours [44]. However, if administered more than seven days after the start of menses, DMPA does not consistently suppress ovulation in the first cycle and a backup method should be used [49]. DMPA IM maintains relatively steady serum concentrations over 12 weeks as it is released from muscle, and then begins to fall. Serum levels are equivalent in obese and normal weight women following initial and subsequent doses of IM DMPA [50]. Following subcutaneous administration, serum levels of MPA are lower in obese women but remain above the threshold for ovulation suppression $(200 \mathrm{pg} / \mathrm{ml})$ for women with a BMI under $40 \mathrm{~kg} / \mathrm{m}^{2}[45,48]$. In one study, a single woman with a BMI over $40 \mathrm{~kg} / \mathrm{m}^{2}$ had serum levels under this threshold but did not ovulate [48]. Women with a BMI over $40 \mathrm{~kg} / \mathrm{m}^{2}$ may have better ovulation suppression with the IM formulation of DMPA, though further research is needed to clarify this relationship.

\section{Obesity-related issues}

Concerns about weight gain are pervasive among users and prescribers of DMPA. However, in studies of adult women there is little evidence that DMPA consistently causes more weight gain than non-hormonal contraceptives. Actual mean weight gain is under $2 \mathrm{~kg}$ over 1-3 years of use for most studies [51], and does not differ based on baseline BMI[52]. DMPA may have a different effect on body composition in adolescents than adults. In one study, normal weight adolescents 
had a 10\% increase in total body fat a 3.4\% decrease in lean body mass over 6 months of DMPA use compared to non-users of hormonal contraception [53].

Weight change with DMPA varies greatly by individual, and weight changes between -7.7 to $21.8 \mathrm{~kg}$ over one year of DMPA use were observed in the Contraceptive CHOICE study [35]. Several predictors for excessive weight gain have been noted, particularly among adolescents. In a retrospective review, obese adolescents who initiated DMPA gained more weight than normal weight adolescents, and gained more weight than adolescents using other contraceptive formulations [52]. Adolescents who gain more than 5\% of their body weight within the first six months of DMPA use continue to have excess weight gain [54]. Finally, African American race is also a predictor of greater weight gain with DMPA[52].

\section{Combined hormonal contraception}

Methods of combined hormonal contraception include oral contraceptive pills (OCPs), the contraceptive vaginal ring (Nuvaring, Merck, Whitehouse Station, NJ, USA), and the contraceptive patch (Ortho Evra, Ortho McNeil Pharmaceutical Inc, Raritan, NJ, USA). These methods contain both an estrogen (ethinyl estradiol, EE) and a progestin component, which provide contraceptive effect primarily through ovulation suppression at the hypothalamic and pituitary level. The inclusion of EE serves to regulate the menstrual cycle and adds to contraceptive effect through suppression of follicle stimulating hormone [55].

\section{Oral contraceptive pills}


OCPs have been the most popular method of contraception in the United States since their introduction in the 1960s [56]. Nearly $82 \%$ of sexually experienced women in the US report use of OCPs at some point in life, and worldwide this ranges from $20-55 \%$ [57]. There are metabolic changes associated with obesity that may alter the pharmacokinetics and efficacy of OCPs, and observational studies have reported conflicting rates of contraceptive failure among obese pill users [58].

In a recent large prospective cohort study of over 52,000 women, OCP failure was reported in two different dosing regimens among normal weight and obese women [59]. Contraceptive failure rates were adjusted for age, parity, and education, and showed a slight but significant increase in failure rates as BMI increased. BMI over 35 was associated with a hazard ratio of 1.5 (95\% CI 1.3-1.8) for contraceptive failure. The prospective cohort Contraceptive CHOICE project had a different conclusion, finding no difference in contraceptive failure by BMI when 1500 users of OCPs, vaginal ring and contraceptive patch were combined [60]. However, the CHOICE project was underpowered for this outcome and the pharmacokinetics of the vaginal ring and contraceptive patch are likely protective given their continuous delivery system.

Dosing strategy of OCPs may be related to risk of failure, especially in obese women. In a comparison of 21 and 24 day dosing of a drosperinone/EE contraceptive pill, 24 day dosing was associated with a lower risk of contraceptive failure for women regardless of BMI [59]. Likewise, a 91 day extended regimen of $20 \mathrm{mcg}$ EE/100mcg levonorgestrel in a population of 1950 women, $25 \%$ of whom 
were obese, showed that pregnancy rates did not vary by weight[61]. A recent pharmacokinetic study demonstrated that continuous dosing of a 20mcg EE/LNG OCP maintained steady state hormone levels better than cyclic dosing in obese women, with superior suppression of the hypothalamic-pituitary-ovarian axis [62]. The longer or more frequent the hormone-free interval, the greater the hormone washout and the greater the chance for follicular recruitment to occur, which could be responsible for contraceptive failure. It is possible that oral contraceptives may be less forgiving of imperfect dosing and more likely to fail with the traditional seven day hormone-free intervals in obese women.

Overall, there is insufficient evidence to conclude that obese women have a higher risk of OCP failure with perfect use compared to normal weight women. Even if there is a tendency towards decreased efficacy with typical use, the absolute risk of failure is still low, and OCPs still offer superior protection to barrier methods [58].

\section{Pharmacokinetics}

The efficacy of OCPs is affected by contraceptive steroid half-life, clearance, time to steady state, and area under the curve, which are all altered in obese women $[63,64]$. Contrary to expectation, volume of distribution does not vary between obese and normal BMI OCP users. Changes in clearance result in a prolonged time to steady state (10 days in obese women compared to 5 in normal weight women), which can lead to a theoretical risk of ovulation and pregnancy in the first 7 days following each hormone-free interval [63]. Subsequent studies demonstrated an 
even longer time to steady state of 13.6 days in obese women [65]. The magnitude of obesity does not correlate linearly with PK alterations. The longer time to reach steady state suggests that obese women will take longer to achieve inhibitory drug concentrations after a hormone-free interval, which might allow a window of OCP failure for follicular recruitment or pregnancy.

It is unclear how these PK differences translate to contraceptive failure in clinical practice, and whether continuous dosing provides better contraceptive effect. OCPs provide additional contraception through cervical mucus thickening, and obese women do not demonstrate changes to cervical mucus scores. Despite differing PK, OCPs retain contraceptive efficacy in most obese women [63,65].

\section{Obesity related issues}

Despite a lack of evidence for weight gain with CHCs, it remains the most common reason cited for discontinuation. A Cochrane review of weight changes of normal weight women taking $\mathrm{CHC}$ found no evidence to support an association between CHC use and weight gain [66]. Individual responses cannot be predicted, but overall there is no definitive evidence for weight gain with CHCs.

\section{Transvaginal and transdermal combined hormonal contraceptives}

The contraceptive vaginal ring (CVR) and contraceptive patch were developed as alternative dosing strategies for combined hormonal contraceptives. The CVR contains EE and etonogestrel and is placed and removed vaginally by the user every 21 days, followed by a 7 day washout. The patch contains EE and 
norelgestromin and is replaced on the skin every 7 days for three weeks, followed by a 7 day washout.

There is minimal data on efficacy of the CVR in obese women. A single underpowered secondary analysis of the Nuvaring phase III trials assessed contraceptive failure in women by body weight [67]. The pregnancy rate did not differ in the highest decile of women (over 167lbs), and there were no method failures in the heaviest women (189-272lbs). Based on this limited data, contraceptive effectiveness does not appear to be diminished in overweight and obese women.

By contrast, a similar secondary analysis of the contraceptive patch phase III trials demonstrated that body weight over $90 \mathrm{~kg}$ is a risk factor for contraceptive failure [68]. Women over $90 \mathrm{~kg}$ represented less than $3 \%$ of the study population, but 33\% of on-treatment pregnancies occurred in these women. This raises concern that the patch is less effective in obese women, though its contraceptive effect is likely still greater than barrier methods alone.

\section{Pharmacokinetics}

When administered vaginally in the CVR, both steroid hormones are rapidly absorbed through vaginal mucosa and reach contraceptive levels within 24 hours [69]. The dosing of both hormones is more stable over time than with OCPs or the patch, and overall EE exposure is less [70]. A single study compared pharmacokinetics of the CVR in obese (BMI $30-40 \mathrm{~kg} / \mathrm{m}^{2}$ ) and normal weight women [71]. Over a single cycle, EE concentrations were lower for obese women $(22 \mathrm{pg} / \mathrm{ml}$ 
vs. $15 \mathrm{pg} / \mathrm{ml}, \mathrm{p}=0.004)$, but ENG levels did not differ $(1,256 \mathrm{pg} / \mathrm{ml}$ vs $1,138 \mathrm{pg} / \mathrm{ml}$, $\mathrm{p}=0.39$ ). Ovulation suppression was equivalent for both groups of women.

There is no published data on pharmacokinetics of the contraceptive patch in obese women, though the initial studies did comment that levels of EE and norelgestromin decreased as body weight increased [68].

\section{Effects on weight}

Weight gain with both the CVR and the patch is minimal and comparable to COCs $[72,73]$. Women with baseline obesity are not at increased risk of weight gain with either method.

\section{Emergency contraception}

Oral emergency contraception refers to formulations which have the potential to prevent pregnancy when taken after unprotected intercourse, primarily through disruption of ovulation. Two formulations are currently available: oral levonorgestrel 1.5mg (Plan B OneStep, Teva Pharmaceuticals), and oral ulipristal acetate $30 \mathrm{mg}$, a selective progestin receptor modulator (UPA) (Ella, HRA Pharma, Paris, France). The copper IUD can also be utilized for emergency contraception when placed within 5 days of unprotected intercourse.

In initial studies of normal weight women, LNG was able to disrupt or delay ovulation $90 \%$ of the time when administered prior to the LH surge and within 72 hours of unprotected intercourse. It has no effect when taken after the LH surge. Its effect is greatest when taken within 24 hours of unprotected intercourse and falls to 
58\% when administered at 72 hours [74]. The strongest predictor of LNG EC failure is body weight, and obese women have a three times greater risk of pregnancy after LNG EC than normal weight women (OR 3.60, 95\% CI 1.96-6.53, $\mathrm{p}<0.001)$. A recent study demonstrated that the efficacy of LNG EC is equivalent to placebo once body weight exceeds $70 \mathrm{~kg}$ (154lbs) or a BMI of $26 \mathrm{~kg} / \mathrm{m}^{2}$ [75].

In a direct comparison trial, oral UPA was more effective at preventing pregnancy than LNG EC, with a pregnancy rate of $1.8 \%$ for UPA and $2.6 \%$ for LNG when each was administered within 72 hours of unprotected intercourse $(p=0.037)$ $[76,77]$. UPA remains more effective for women with higher body weights, but its effect also falls to that of placebo when body weight exceeds $88 \mathrm{~kg}$ (194lb) or a BMI of $35 \mathrm{~kg} / \mathrm{m}^{2}[75]$. These data are concerning that both formulations of oral EC may be less effective or ineffective in obese women, and further data are needed to elucidate this effect. Importantly, the pharmacokinetics of these drugs are not linear and simply increasing the dose is unlikely to achieve contraceptive effect. The copper IUD remains the most effective option for EC with a pregnancy rate of $<0.1 \%$, and should not lose efficacy in obese women $[78,79]$.

\section{Pharmacokinetics}

The contraceptive effect of oral EC is provided by rapidly achieving a serum level to block the LH surge. Obese women are known to have a longer time to achieve steady state and lower serum levels when administered COCs containing LNG. It is possible that the lower efficacy of LNG EC is related to these altered 
pharmacokinetics and an inability to reach contraceptive threshold after a single dose [65]. Pharmacokinetics of UPA in obese women remain unpublished.

\section{Practice points}

- Sexual behaviors and contraceptive use do not appear to vary by BMI in adult women, though obese adolescents may be at higher risk of high-risk sexual behavior and unintended pregnancy compared to normal weight teens.

- LARCs and permanent contraception are the most effective options for all women regardless of BMI, and can be used safely by obese women.

- Depo Provera, oral contraceptive pills and the contraceptive vaginal ring appear to have equivalent contraceptive effect in obese women. The contraceptive patch appears to have decreased efficacy in women over $90 \mathrm{~kg}$, but still confers some contraceptive protection.

- Oral emergency contraception (LNG and UPA) lose effectiveness in obese women. The copper IUD remains the most effective method of EC for all women.

\section{Research agenda}

- Further clarification of the pharmacokinetics and pharmacodynamics of contraceptive implants in obese women to ensure equivalent contraceptive effect in various body weights.

- Development of a regimen of oral emergency contraception to suppress ovulation in overweight and obese women. 


\section{References}

* designates a key reference

1. Ogden C, Carroll M, Kit B, Flegal K. Prevalence of Obesity in the United States, 2009-2010. National Center for Health Statistics. 2012 Jan 9;82:1-8.

2. Obesity and overweight [Internet]. World Health Organization. [cited 2013 Mar 6]. Available from: http://www.who.int/mediacentre/factsheets/fs311/en/index.html

3. Prentice A. The emerging epidemic of obesity in developing countries. International Journal of Epidemiology. 2005 Jul 4;35(1):93-9.

4. Weiss JL, Malone FD, Emig D, Ball RH, Nyberg DA, Comstock CH, et al. Obesity, obstetric complications and cesarean delivery rate-a population-based screening study. American Journal of Obstetrics and Gynecology. 2004 Apr;190(4):1091-7.

5. Phelan S. Pregnancy: a "teachable moment" for weight control and obesity prevention. American Journal of Obstetrics and Gynecology. Elsevier Inc; 2010 Feb 1;202(2):135.e1-135.e8.

6. Nagelkerke N, Bernsen R, Sgaier S, Jha P. Body mass index, sexual behaviour, and sexually transmitted infections: an analysis using the NHANES 1999-2000 data. BMC Public Health. 2006;6(1):199. 
7. Leech TGJ, Dias JJ. Risky Sexual Behavior: A Race-specific Social Consequence of Obesity. J Youth Adolescence. 2011 May 4;41(1):41-52.

8. Lowry R, Robin L, Kann L, Galuska DA. Associations of Body Mass Index with Sexual Risk-Taking and Injection Drug Use among US High School Students. Journal of Obesity. 2014;2014(3):1-10.

9. Bajos N, Wellings K, Laborde C, Moreau C, for the CSF Group. Sexuality and obesity, a gender perspective: results from French national random probability survey of sexual behaviours. BMJ. 2010 Jun 15;340.

* 10. Kaneshiro B, Jensen JT, Carlson N, Harvey SM, Nichols M, Edelman A. Body Mass Index and Sexual Behavior. Obstetrics \& Gynecology. 2008 Aug 13;112(3):586-92.

11. Gesink Law DC, Maclehose RF, Longnecker MP. Obesity and time to pregnancy. Human Reproduction. 2006 Sep 27;22(2):414-20.

12. van der Steeg JW, Steures P, Eijkemans MJC, Habbema JDF, Hompes PGA, Burggraaff JM, et al. Obesity affects spontaneous pregnancy chances in subfertile, ovulatory women. Human Reproduction. 2007 Dec 14;23(2):3248.

13. Sim KA, Partridge SR, Sainsbury A. Does weight loss in overweight or obese women improve fertility treatment outcomes? A systematic review. Obes Rev. 2014 Aug 6;15(10):839-50. 
14. National Center for Health Statistics. Current Contraceptive Use in the United States, 2006-2010, and Changes in Patterns of Use Since 1995. Jones J, Mosher WD, Daniels K, editors. 2012 Oct 9;:1-26.

15. World Contraceptive Use 2009 [Internet]. United Nations Population Division, Fertility and Family Planning Section. [cited 2014 Aug 25]. Available from: http://www.un.org/esa/population/publications/WCU2009/Main.html

16. Vahratian A, Barber JS, Lawrence JM, Kim C. Family-Planning Practices Among Women With Diabetes and Overweight and Obese Women in the 2002 National Survey for Family Growth. Diabetes Care. 2009 May 21;32(6):102631.

17. Schraudenbach A, McFall S. Contraceptive use and contraception type in women by body mass index category. Women's Health Issues. Jacobs Institute of Women's Health; 2009 Nov 12;19(6):381-9.

18. Callegari LS, Nelson KM, Arterburn DE, Prager SW, Schiff MA, Schwarz EB. Factors associated with lack of effective contraception among obese women in the United States. Contraception. Elsevier B.V; 2014 Jun 16;:1-7.

* 19. Winner B, Peipert JF, Q Z, C B, Madden TE, Allsworth JE, et al. Effectiveness of Long-Acting Reversible Contraception. New England Journal of Medicine. 2012 May 15;366(21):1998-2007.

20. Westhoff CL, Torgal AT, Mayeda ER, Shimoni N, Stanczyk FZ, Pike MC. 
Predictors of noncompliance in an oral contraceptive clinical trial.

Contraception. Elsevier Inc; 2012 May 1;85(5):465-9.

21. Finer LB, Zolna MR. Unintended pregnancy in the United States: incidence and disparities, 2006. Contraception. Elsevier Inc; 2011 Nov 1;84(5):478-85.

22. Kaneshiro B, Edelman A, Carlson N, Nichols M, jensen J. The relationship between body mass index and unintended pregnancy: results from the 2002 National Survey of Family Growth. Contraception. 2008 Apr;77(4):234-8.

23. Chan LM, Westhoff CL. Tubal sterilization trends in the United States. Fertility and Sterility. Elsevier Ltd; 2010 Jun 1;94(1):1-6.

24. Fernandez H, Legendre G, Blein C, Lamarsalle L, Panel P. Tubal sterilization: pregnancy rates after hysteroscopic versus laparoscopic sterilization in France, 2006. European Journal of Obstetrics and Gynecology. Elsevier Ireland Ltd; 2014 Jun 29;:1-5.

25. Trussell J. Contraceptive failure in the United States. Contraception. Elsevier Inc; 2011 May 1;83(5):397-404.

26. Peterson H, Trussell J, Hughes E, Taylor L. The risk of pregnancy after tubal sterilization: Findings from the US Collaborative Review of Steriliation (CREST). American Journal of Obstetrics and Gynecology. 1996 Aug 18;174(4):1161-9.

27. Rodriguez MI, Seuc A, Sokal DC. Comparative efficacy of postpartum 
sterilisation with the titanium clip versus partial salpingectomy: a randomised controlled trial. BJOG: An International Journal of Obstetrics \& Gynaecology. 2012 Aug 24;120(1):108-12.

28. Creinin MD, Zite N. Female tubal sterilization:. Obstetrics \& Gynecology. $124(3)$.

29. Anderson T, Yunker A, Scheib S, Callahan T. Hysteroscopic Sterilization Success in Outpatient vs Office Setting Is Not Affected by Patient or Procedural Characteristics. The Journal of Minimally Invasive Gynecology. Elsevier Ltd; 2013 Jul 22;e-pub ahead of print:1-6.

30. Gariepy AM, Creinin MD, Smith KJ, Xu X. Probability of pregnancy after sterilization: a comparison of hysteroscopic versus laparoscopic sterilization. Contraception. Elsevier Inc; 2014 Aug 1;90(2):174-81.

* 31. Xu H, Wade JA, Peipert JF, Zhao Q, Madden T, Secura GM. Contraceptive Failure Rates of Etonogestrel Subdermal Implants in Overweight and Obese Women. Obstetrics \& Gynecology. 2012 Jul;120(1):21-6.

32. Vilos GA, Marks J, Tureanu V, Abu-Rafea B, Vilos AG. The Levonorgestrel Intrauterine System Is an Effective Treatment in Selected Obese Women with Abnormal Uterine Bleeding. The Journal of Minimally Invasive Gynecology. Elsevier Ltd; 2011 Jan 2;18(1):75-80.

33. Curtis KM, Marchbanks PA, Peterson HB. Neoplasia with use of intrauterine 
devices. Contraception. 2007 Jun;75(6):S60-9.

34. Ronnerdag M, Odlind V. Health effects of long-term use of the intrauterine levonorgestrel-releasing system. Acta Obst Gynecol Scand. 1999 Aug $4 ; 78: 716-21$

*35. Vickery Z, Madden T, Zhao Q, Secura GM, Allsworth JE, Peipert JF. Weight change at 12 months in users of three progestin-only contraceptive methods. Contraception. Elsevier Inc; 2013 Oct 1;88(4):503-8.

36. Sivin I, Alvarez F, Mishell D Jr, Darney PD, Wan L, Brache V, et al. Contraception With Two Levonorgestrel Rod Implants. Contraception. 1998 Dec 8;58:275-82.

*37. Mornar S, Chandra A, Mistretta S, Neustadt A, Martinez G, Gilliam M. Pharmacokinetics of the etonogestrel contraceptive implant in obese women. American Journal of Obstetrics and Gynecology. Elsevier Inc; 2012 Aug 1;207(2):110.e1-110.e6.

38. Huber J. Pharmacokinetics of Implanon. Contraception. 1998 Jan 22;58:85S$90 \mathrm{~S}$.

39. Diaz S, Pavez M, Moo-Young C, Bardin CW, Croxatto HB. Clinical trial with 3keto-desogestrel subdermal implants. Contraception. 1991 Mar 4;44(4):393408.

40. Sivin I, Wan L, Ranta S, Alvarez F, Brache V, Mishell D Jr, et al. Levonorgestrel 
concentrations during 7 years of continuous use of Jadelle contraceptive implants. Contraception. 2001 Aug 17;64:43-9.

41. Makarainen L, van Beek A, Tuomivaara L, Asplund B, Bennick H. Ovarian function during the use of a single contraceptive implant: Implanon compared with Norplant. Fertility and Sterility. 1998 Mar 19;69(4):714-21.

42. Davies G, Feng LX, Newton JR. Release characteristics, ovarian activity and menstrual bleeding pattern with a single contraceptive implant releasing 3keodesogestrel. Contraception. 1993 Mar 4;47:251-61.

43. Croxatto H, Urbancsek J, Massai MR, Bennick H, van Beek A. A multicentre efficacy and safety study of the single contraceptive implant Implanon. Human Reproduction. 1999 Mar 26;14(4):976-81.

44. Mishell D Jr. Pharmacokinetics of depot medroxyprogesterone acetate contraception. Journal of Reproductive Medicine. 1996 May 29;51(S5):38190.

45. Jain J, Jakimiuk AJ, Bode FR, Ross D, Kaunitz AM. Contraceptive efficacy and safety of DMPA-SC. Contraception. 2004 Oct;70(4):269-75.

46. Fotherby K, Koetsawang S. Metabolism of injectable formulations of contraceptive steroids in obese and thin women. Contraception. 1982 Jan $4 ; 26(1): 51-8$.

47. Benagiano G, Diczfalusy E, Goldzieher JW, Gray R. Multinational comparative 
clinical evaluation of two long-acting contraceptive steroids: Norethisterone oenanthate and medroxyprogesterone acetate. Contraception. 1977 May $4 ; 15(5): 513-33$.

48. Segall-Gutierrez P, Taylor D, Liu X, Stanzcyk F, Azen S, Mishell DR Jr. Follicular development and ovulation in extremely obese women receiving depomedroxyprogesterone acetate subcutaneously. Contraception. Elsevier Inc; 2010 Jun 1;81(6):487-95.

49. Siriwongse T, Snidvongs W, Tantayaporn P, Leepipatpaiboon S. Effect of DepoMedroxyprogesterone Acetate on Serum Progesterone Levels When Administered on Various Cycle Days. Contraception. 1982 Nov 4;26(5):48793.

50. Smit J, Botha J, McFadyen L, Beksinska M. Serum medroxyprogesterone acetate levels in new and repeat users of depot medroxyprogesterone acetate at the end of the dosing interval. Contraception. 2004 Jan;69(1):3-7.

51. Lopez LM, Edelman A, Chen M, Otterness C, Trussell J, Helmerhorst FM. Progestin-only contraceptives: effects on weight (Review). Cochrane Database. 2012 Dec 31;:1-68.

52. Gerlach LS, Saldaña SN, Wang Y, Nick TG, Spigarelli MG. Retrospective Review of the Relationship Between Weight Change and Demographic Factors Following Initial Depot Medroxyprogesterone Acetate Injection in Adolescents. Clinical Therapeutics. Elsevier Inc; 2011 Feb 1;33(2):182-7. 
53. Bonny AE, Secic M, Cromer B. A Longitudinal Comparison of Body Composition Changes in Adolescent Girls Receiving Hormonal Contraception. Journal of Adolescent Health. Elsevier Ltd; 2009 Oct 1;45(4):423-5.

54. Bonny AE, Secic M, Cromer B. Early Weight Gain Related to Later Weight Gain in Adolescents on Depot Medroxyprogesterone Acetate. Obstetrics \& Gynecology. 2011 Apr;117(4):793-7.

55. Fritz MA, Speroff L. Clinical gynecologic endocrinology and infertility. 8 ed. Lippincott Williams \& Wilkins; 2010.

56. Mosher WD, Martinez G, Chandra A, Abma J, Wilson SJ. Use of Contraception and Use of Family Planning Services in the United States: 1982-2002. Center for Disease Control Advance Data. 2004 Dec 6;(350):1-46.

57. Townsend JW, Sitruk-Ware R, Williams K, Askew I, Brill K. New strategies for providing hormonal contraception in developing countries. Contraception. Elsevier Inc; 2011 May 1;83(5):405-9.

58. Trussell J, Schwarz EB, Guthrie K. Obesity and oral contraceptive pill failure. Contraception. Elsevier Inc; 2009 May 1;79(5):334-8.

* 59. Dinger J, Do Minh T, Buttmann N, Bardenheuer K. Effectiveness of Oral Contraceptive Pills in a Large U.S. Cohort Comparing Progestogen and Regimen. Obstetrics \& Gynecology. 2011 Jan;117(1):33-40.

* 60. McNicholas C, Zhao Q, Secura G, Allsworth JE, Madden T, Peipert JF. 
Contraceptive Failures in Overweight and Obese Combined Hormonal Contraceptive Users. Obstetrics \& Gynecology. 2013 Mar;121(3):585-92.

61. Westhoff C, Reape K, Shu H. Impact of body weight on observed pregnancy rates with a low-dose estrogen, 91-day extended regimen oral contraceptive. Contraception. Elsevier B.V; 2009 Aug 1;80(2):196-7.

62. Edelman AB, Cherala G, Munar MY, McInnis M, Stanczyk FZ, Jensen JT. Correcting oral contraceptive pharmacokinetic alterations due to obesity: a randomized controlled trial. Contraception. Elsevier Inc; 2014 Jul 24;:1-7.

63. Edelman AB, Carlson NE, Cherala G, Munar MY, Stouffer RL, Cameron JL, et al. Impact of obesity on oral contraceptive pharmacokinetics and hypothalamic. Contraception. Elsevier Inc; 2009 Aug 1;80(2):119-27.

*64. Edelman AB, Cherala G, Stanczyk FZ. Metabolism and pharmacokinetics of contraceptive steroids in obese women: a review. Contraception. Elsevier Inc; 2010 Oct 1;82(4):314-23.

*65. Edelman AB, Cherala G, Munar MY, DuBois B, McInnis M, Stanczyk FZ, et al. Prolonged monitoring of ethinyl estradiol and levonorgestrel levels confirms an altered pharmacokinetic profile in obese oral contraceptives users. Contraception. Elsevier Inc; 2013 Feb 1;87(2):220-6.

66. Gallo MF, Lopez LM, Schulz KF, Helmerhorst FM. Combination contraceptives: effects on weight (Review). Cochrane Database. 2012 Dec 31;:1-116. 
67. Westhoff C. Higher Body Weight Does Not Affect NuvaRing's Efficacy (abstract). Obstetrics \& Gynecology. 2005 Mar 15;105(4S):56S.

68. Zieman M, Guillebaud J, Weisberg E, Shangold G, Fisher AC, Creasy G. Contraceptive efficacy and cycle control with the Ortho EvraTM/EvraTM transdermal system: the analysis of pooled data. Fertility and Sterility. 2002 Jan 30;77(2):S13-8.

69. Timmer C, Mulders TM. Pharmacokinetics of Etonogestreland Ethinylestradiol Released from a Combined Contraceptive Vaginal Ring. Clinical Pharmacokinetics. 2000 Sep 29;39(3):233-42.

70. van den Heuvel MW, van Bragt AJM, Alnabawy AKM, Kaptein MCJ. Comparison of ethinylestradiol pharmacokinetics in three hormonal contraceptive formulations: the vaginal ring, the transdermal patch and an oral contraceptive. Contraception. 2005 Sep;72(3):168-74.

71. Westhoff CL, Torgal AH, Mayeda ER, Petrie K, Thomas T, Dragoman M, et al. Pharmacokinetics and ovarian suppression during use of a contraceptive vaginal ring in normal-weight and obese women. American Journal of Obstetrics and Gynecology. Elsevier Inc; 2012 Jul 1;207(1):39.e1-39.e6.

72. O'Connell KJ, Osborne LM, Westhoff C. Measured and reported weight change for women using a vaginal contraceptive ring vs. a low-dose oral contraceptive. Contraception. 2005 Nov;72(5):323-7. 
73. Sibai B, Odlind V, Meador M, Shangold G, Fisher AC, Creasy G. A comparative and pooled analysis of the safety and tolerability of the contraceptive patch (Ortho EvraTM/EvraTM). Fertility and Sterility. 2002 Jan 30;77(2):S19-S26.

74. Croxatto HB, HA B, Pavez M, Cochon L, Forcelledo ML, Alvarez F, et al. Pituitary-ovarian function following the standard levonorgestrel emergency contraceptive dose or a single 0.75 -mg dose given on the days preceding ovulation. Contraception. 2004 Dec;70(6):442-50.

* 75. Glasier A, Cameron ST, Blithe D, Scherrer B, Mathe H, Levy D, et al. Can we identify women at risk of pregnancy despite using emergency contraception? Data from randomized trials of ulipristal acetate and levonorgestrel. Contraception. Elsevier Inc; 2011 Mar 28;:1-5.

76. Glasier AF, Cameron ST, Fine PM, Logan SJ, Casale W, Van Horn J, et al. Ulipristal acetate versus levonorgestrel for emergency contraception: a randomised non-inferiority trial and meta-analysis. The Lancet. Elsevier Ltd; 2010 Feb 13;375(9714):555-62.

77. Moreau C, Trussell J. Results from pooled Phase III studies of ulipristal acetate for emergency contraception. Contraception. Elsevier Inc; 2012 Dec $1 ; 86(6): 673-80$.

78. Cleland K, Zhu H, Goldstuck N, Cheng L, Trussell J. The efficacy of intrauterine devices for emergency contraception: a systematic review of 35 years of experience. Human Reproduction. 2012 Jun 15;27(7):1994-2000. 
79. Turok DK, Godfrey EM, Wojdyla D, Dermish A, Torres L, Wu SC. Copper T380 intrauterine device for emergency contraception: highly effective at any time in the menstrual cycle. Human Reproduction. 2013 Oct 14;28(10):2672-6. 


\begin{tabular}{|c|c|c|c|c|}
\hline $\begin{array}{l}\text { Guidelines for } \\
\text { obese women } \\
\left(\mathrm{BMI}>30 \mathrm{~kg} / \mathrm{m}^{2}\right)\end{array}$ & $\begin{array}{l}\text { Copper IUD/ } \\
\text { LNG-IUS }\end{array}$ & $\begin{array}{l}\text { ENG or LNG } \\
\text { implant }\end{array}$ & DMPA & $\begin{array}{l}\text { Combined } \\
\text { (OCPs, patch, } \\
\text { ring) }\end{array}$ \\
\hline $\begin{array}{l}\text { Medical Eligibility } \\
\text { Criteria for } \\
\text { Contraceptive Use } \\
\text { (World Health } \\
\text { Organization) } 2009\end{array}$ & 1 & 1 & $\begin{array}{c}1 \\
(2 \text { in } \\
\text { adolescents } \\
<\text { age } 18)\end{array}$ & 2 \\
\hline $\begin{array}{l}\text { UK Medical Eligibility } \\
\text { Criteria for } \\
\text { Contraceptive Use } \\
\text { (Royal College of } \\
\text { Obstetricians and } \\
\text { Gynaecologists, } \\
\text { Faculty of Sexual } \\
\text { and Reproductive } \\
\text { Health Care) } 2009\end{array}$ & 1 & 1 & 1 & $\begin{array}{l}\mathrm{BMI} 30-34 \\
\mathrm{~kg} / \mathrm{m}^{2}: 2 \\
\mathrm{BMI}>35 \\
\mathrm{~kg} / \mathrm{m}^{2}: 3\end{array}$ \\
\hline
\end{tabular}

Table 1. Recommendations for the use of contraception in obese women. $1=\mathrm{A}$ condition for which there is no restriction for use. $2=$ A condition for which the advantages of using the method generally outweigh the theoretical or proven risks. $3=$ A condition for which the theoretical or proven risks usually outweigh the advantages of using the method. $4=$ A condition that represents an unacceptable health risk if the contraceptive method is use. 